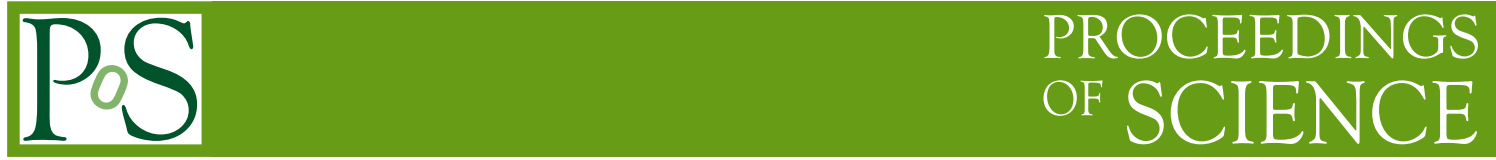

\title{
Quantum Gravity phenomenology constraints potentialities of next space gamma-ray experiments
}

\author{
Aldo Morselli* \\ INFN Roma2, Italy \\ E-mail: aldo.morselli@roma2.infn.it
}

The next space gamma-ray experiments like AGILE and GLAST could in principle give some signals of new physics with the discovery of gamma rays from the annihilation of dark matter particles or Lorentz invariance violation effects in transient sources. Here we give an update of the status of these experiments and these searches.

From Quantum to Emergent Gravity: Theory and Phenomenology

June 11-15 2007

Trieste, Italy

${ }^{*}$ Speaker. 
Table 1: Space experiments: Technology and Physics

\begin{tabular}{llll}
\hline$*$ & Cosmic rays & & \\
\hline$\sqrt{ }$ & SilEye-1 & MIR & $1995-1997$ \\
$\sqrt{ }$ & SilEye-2 & MIR & $1997-2001$ \\
$\sqrt{ }$ & AMS-01 & Shuttle & 1998 \\
$\sqrt{ }$ & NINA-1 & Resurs & 1998 \\
$\sqrt{ }$ & NINA-2 & MITA & 2000 \\
$\sqrt{ }$ & Alteino (SilEye-3) & ISS & 2002 (April 25) \\
$\sqrt{ }$ & PAMELA & ResursDK1 & 2006 (June 15) \\
$\triangleright$ & AMS-02 & ISS & $2009 ?$ \\
$*$ & Low energy gamma-ray & & \\
\hline$\sqrt{ }$ & HETE-2 & Pegasus & 2002 (Jan 14) \\
$\sqrt{ }$ & INTEGRAL & Proton & 2002 (Oct 17) \\
$\sqrt{ }$ & SWIFT & Delta II & 2004 (Nov. 20) \\
$\sqrt{ }$ & AGILE & MITA & 2007 (April 23) \\
$\triangleright$ & GLAST & Delta II & 2008 (May) \\
\hline$*$ & Ultra high energy cosmic rays & & \\
\hline$\triangleright$ & JEM-EUSO & ISS & 2013 \\
\hline \hline$*$ & Gravitational wave & & \\
\hline$\triangleright$ & LISA Pathfinder & Soyuz \\
$\triangleright$ & LISA & Delta II & $2015 ?$ \\
\hline
\end{tabular}

\section{Introduction}

There is a lot of activity nowadays in space. A list of experiments is given in table 1. HETE-2 (High Energy Transient Explorer-2), INTEGRAL (INTernational Gamma-Ray Astrophysics Laboratory) and Swift are for gamma-rays with energies respectively $0.5-400 \mathrm{KeV}, 0,2-150 \mathrm{KeV}, 15$ $\mathrm{KeV}-10 \mathrm{MeV}$. AGILE (Astrorivelatore Gamma a Immagini LEggero or Extremely Light Imager for Gamma-ray Astronomy) and GLAST (Gamma-Ray Large Area Space Telescope) are for high energy gamma-rays (20 MeV-50 GeV and $20 \mathrm{MeV}-300 \mathrm{GeV}$ respectively), PAMELA (a Payload for Antimatter Matter Exploration and Light-nuclei Astrophysics) and AMS (Alpha Magnetic Spectrometer) will investigate the high energy cosmic rays (up to TeV) and EUSO (Extreme Universe Space Observatory) will collect thousand events/year of Cosmic Rays at E $>10^{20} \mathrm{eV}$. For gravitational wave the LISA Pathfinder mission is foreseen at the end of 2009 and will test the technology needed to develop the LISA (Laser Interferometry Space Antenna) mission, foreseen in 2015-2020.

In this paper we will not address the issue of the gravitational wave missions, but we will focus on how high energy photons and cosmic ray missions could be useful to General Relativity and Gravitational Physics.

As we will see in more details, high energy photons can be useful for several reasons:

1) they can bring a better knowledge of possible gravitational wave sources ( Pulsars, AGNs, SNs, GRBs, Black Holes....)

2) to examine Lorenz invariance through tests of the velocity of light dispersion laws or modification of Infra-red cut-off 
3) to verify cosmological models through tests of consistence on the Hubble constant or on cosmological models with Dark Matter

In section 2 we will describe the first point, in section 3 we will examine points 2 and 3 and in section 4 and 5 we will give a brief description of the AGILE and GLAST experiments.

\section{Better knowledge of possible gravitational wave sources}

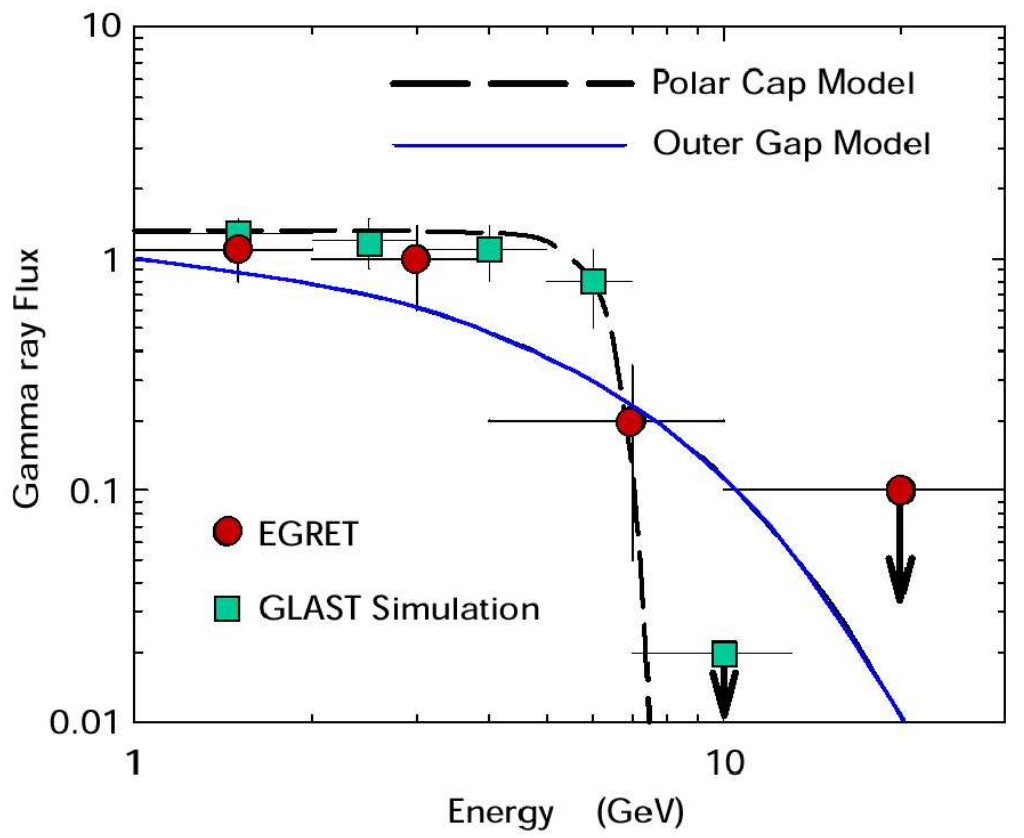

Figure 1: Modeled high-energy pulsar spectrum, showing the improvement in resolution between EGRET and GLAST. The polar cap model predicts a sharp high-energy cutoff, while the outer gap model predicts a more gradual cutoff. Unlike EGRET, GLAST will be able to distinguish the true shape of the spectrum (the polar gap model is assumed in this GLAST simulation).

\subsection{Pulsars}

Pulsars, magnetized neutron stars whose rotation produces the characteristic pulsation, are unparalleled physics laboratories for many applications; nevertheless, some of the intrinsic feature of pulsars remain unknown: How and where does the particle acceleration take place? What is the shape of the particle beam, and how is this energy converted to radiation? Where does the bulk of the energy go, since it is not seen in radiation? Except for transients, pulsars are the brightest sources in the gamma-ray sky. Only in gamma rays a significant fraction of the pulsar spin-down luminosity can be seen. Pulsar models have changed dramatically to explain the gammaray observations but up to now among the $\sim 1500$ rotation-powered pulsars, observed mainly in the radio band, only 7 are seen as pulsed gamma-ray sources, and another $\sim 10$ have been reported as possible detections [1]. The availability of a wider and deeper sample of gamma-ray observations of pulsars could set better constraints on emission models that predict different beaming angles and directions for the radio and gamma-ray emission. In particular the two primary models proposed 
to explain particle acceleration and gamma-ray generation: the outer gap model [3], where particle acceleration occurs in the outer magnetosphere of the pulsar and the polar cap model [2], where particle acceleration occurs near the magnetic polar cap of the pulsar, predicts different spectral breaks (see figure 1) that can be seen by AGILE or GLAST.

Another possibility is to look for microstructures in gamma-ray light curves such as those clearly seen in single radio pulses from Vela [4] that are far from being understood.

It is now possible to see such structures because the deadtime of the gamma ray missions is constantly decreasing. In figure 2 is shown the deadtime history in function of time. The spark chambers limited the EGRET deadtime to $\sim 200 \mathrm{~ms}$; the use of silicon detectors in the next experiments will allow the breakdown seen in figure 2 of a factor more than $10^{3}$, allowing the studies mentioned and several new ones that we are going to mention.

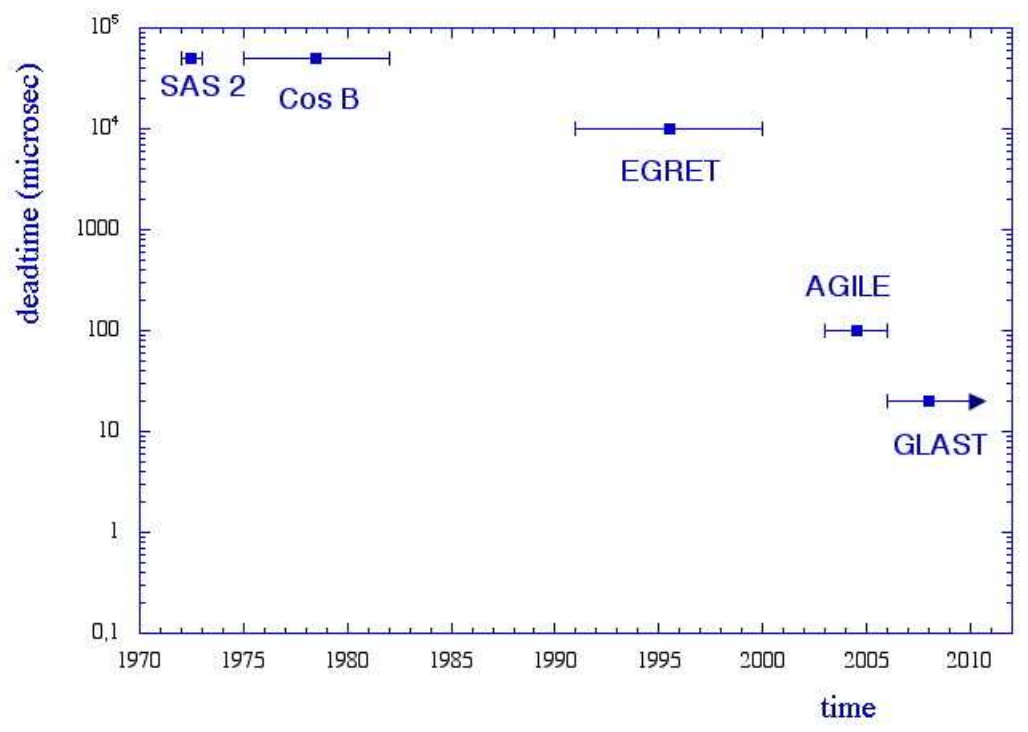

Figure 2: Gamma -ray missions deadtime

\subsection{Active Galactic Nuclei's}

Before EGRET, 3C 273 was the only active galactic nucleus (AGN) known to emit high-energy gamma rays. With the detection of more than 60 AGN, EGRET has strengthened the unified model of AGN as supermassive black holes with accretion disk and jets and we now known that there is an entire class of active galaxies that probably represent the largest class of high energy gamma-ray emitters: the blazars. Blazars are flat radio spectrum AGN whose members include BL Lac objects and highly polarized and optically violently variable quasars that often emit more in gamma-rays than in any other frequencies.

\section{Lorenz invariance test}

\subsection{The blackbody background attenuation}

It is well known that the gamma ray fluxes from high energy sources can loose photons from the interaction with the blackbody background through the process $\gamma \gamma \rightarrow e^{+} e^{-}$(see figure 3). The 


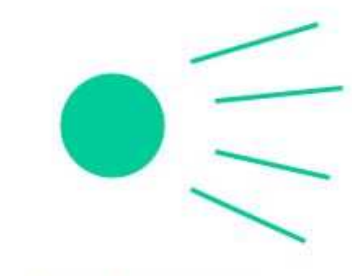

$\gamma$ ray source
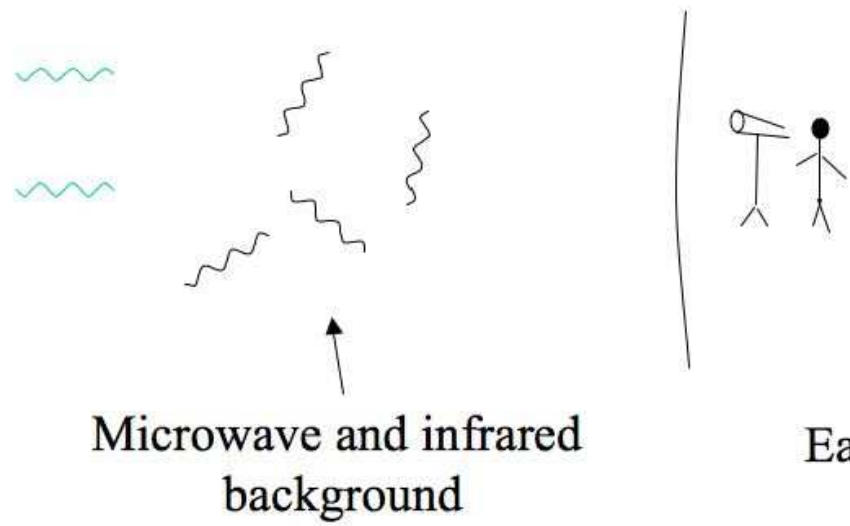

Figure 3: Flux absorption due to the interaction with the infrared and microwave background

electrons then loose the source direction due to the interstellar magnetic field. The cross section of the process is [5]

$$
\sigma_{\gamma \gamma}=\frac{\pi r_{e}^{2}}{2}\left(1-v^{2}\right)\left\{\left(3-v^{4}\right) \ln \left(\frac{1+v}{1-v}\right)-2 v\left(2-v^{2}\right)\right\}
$$

where $r_{e}$ is the classical radius of the electron and

$$
v=\sqrt{1-\frac{4 m_{e}^{2}}{2 \omega_{1} \omega_{2}(1-\cos \vartheta)}}
$$

$\omega_{1}$ e $\omega_{2}$ are the energy of the blackbody spectrum and the high energy gamma ray respectively and $\vartheta$ is their incidence angle.

Because the blackbody spectrum is isotropic, we can integrate in $d \Omega=2 \pi \sin \vartheta d \vartheta$ with the normalization $1 /(4 \pi)$, then the ratio between the flux $\mathrm{I}(\mathrm{L})$ at a distance $\mathrm{L}$ from the source and the initial flux I can be written as:

$$
I / I_{0}=\exp \left(-k_{\gamma} \cdot L\right)
$$

where $k_{\gamma}$ is the absorption coefficient

$$
k_{\gamma}=\frac{1}{2} \int_{0}^{\infty} \int_{\vartheta *}^{\pi} \frac{d n_{\gamma}}{d \omega_{1}} \sigma_{\gamma \gamma} \sin \vartheta d \vartheta d \omega_{1}
$$

and $d n_{\gamma} / d \omega_{1}$ is the Plank black body distribution

$$
d n_{\gamma} / d \omega_{1}=\frac{1}{\hbar^{3} c^{3} \pi^{2}} \frac{\omega_{1}^{2}}{\exp \left(\omega_{1} / k T\right)-1}
$$

The minimum angle $\vartheta^{*}$ depends on photon energy because the energy in the center of mass must be

$$
\sqrt{2 \omega_{1} \omega_{2}(1-\cos \vartheta)} \geq 2 m_{e}
$$




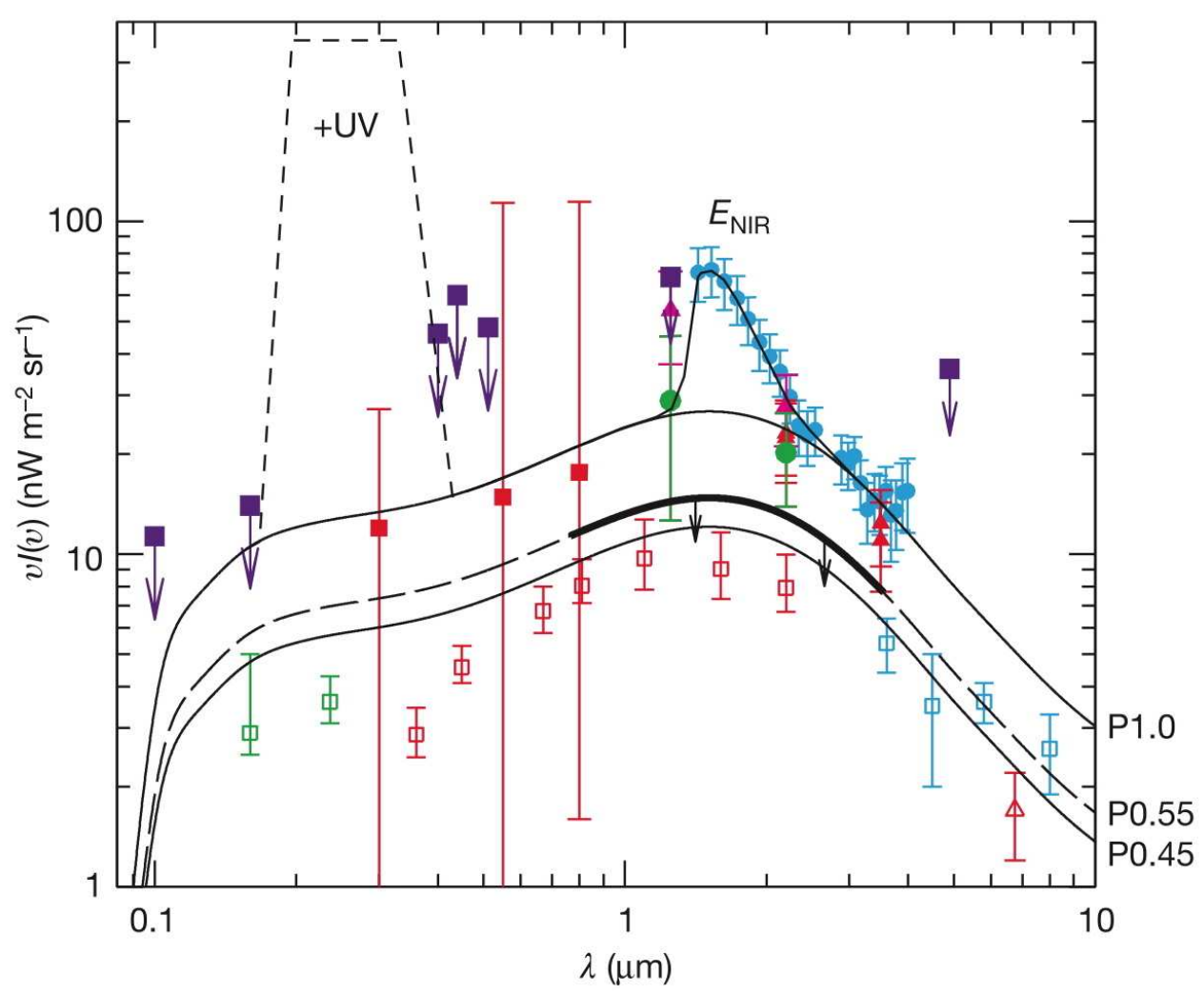

Figure 4: Diffuse extragalactic background radiation. The reported fluxes at far infrared wavelengths above $60 \mu \mathrm{m}$, as well as at optical wavelengths below $1 \mu \mathrm{m}$ are taken from the recent review by [7]. Open symbols correspond to the integrated light from galaxy counts, and thus must be considered lower limits for the Extragalactic Background Light (EBL) Direct measurements are shown as filled symbols. The curves show the EBL shapes used to reconstruct the intrinsic spectra of the blazars measured by HESS [6]

\subsection{The Infrared radiation field}

A similar effect occurs at lower gamma-ray energies for the interaction with the Infrared radiation field ( because of the higher energies of infrared photons compared to the microwave ones. Observational determinations of the extragalactic infrared background are plagued by the difficulty of separating the true extragalactic component from Galactic radiation and instrumental background. A summary of the observations is shown in figure 4 . Using the curves in figure 4 one can calculate the factor $\exp [-\tau(E)]=\exp \left(-k_{\gamma} \cdot L\right)$, i.e. the degree of absorption for different $z$ as shown in figure 5 (on the left). It is interesting to notice at this point that for any reasonable infrared background model there is a strong modification of the original spectral shape. Reasons are the strong energy dependence of the mean free path of $\gamma$-rays in the intergalactic medium, $\Lambda(E)$, and the fact that the optical depth $\tau=c z H_{0}^{-1} \Lambda^{-1}(E)$ significantly exceeds unity at all $\gamma$-ray energies above $300 \mathrm{GeV}$ for $\mathrm{H} 1426+428(z=0.129)$. In figure 6 it is shown the mean free path of the Universe due to the interaction with both the infrared and microwave backgrounds. In figure 5 (on the right) it is shown the spectrum of $\mathrm{H} 1426+428$ (data and possible fit assuming initial power law spectrum $\propto E^{-1.9}$ as suggested by the X-ray spectrum, which extends up to $100 \mathrm{keV}$ ). The conclusion is that the detection of $\mathrm{TeV} \gamma$-rays from $\mathrm{H} 1426+428$ should lead to a revision of the current conceptual view of 

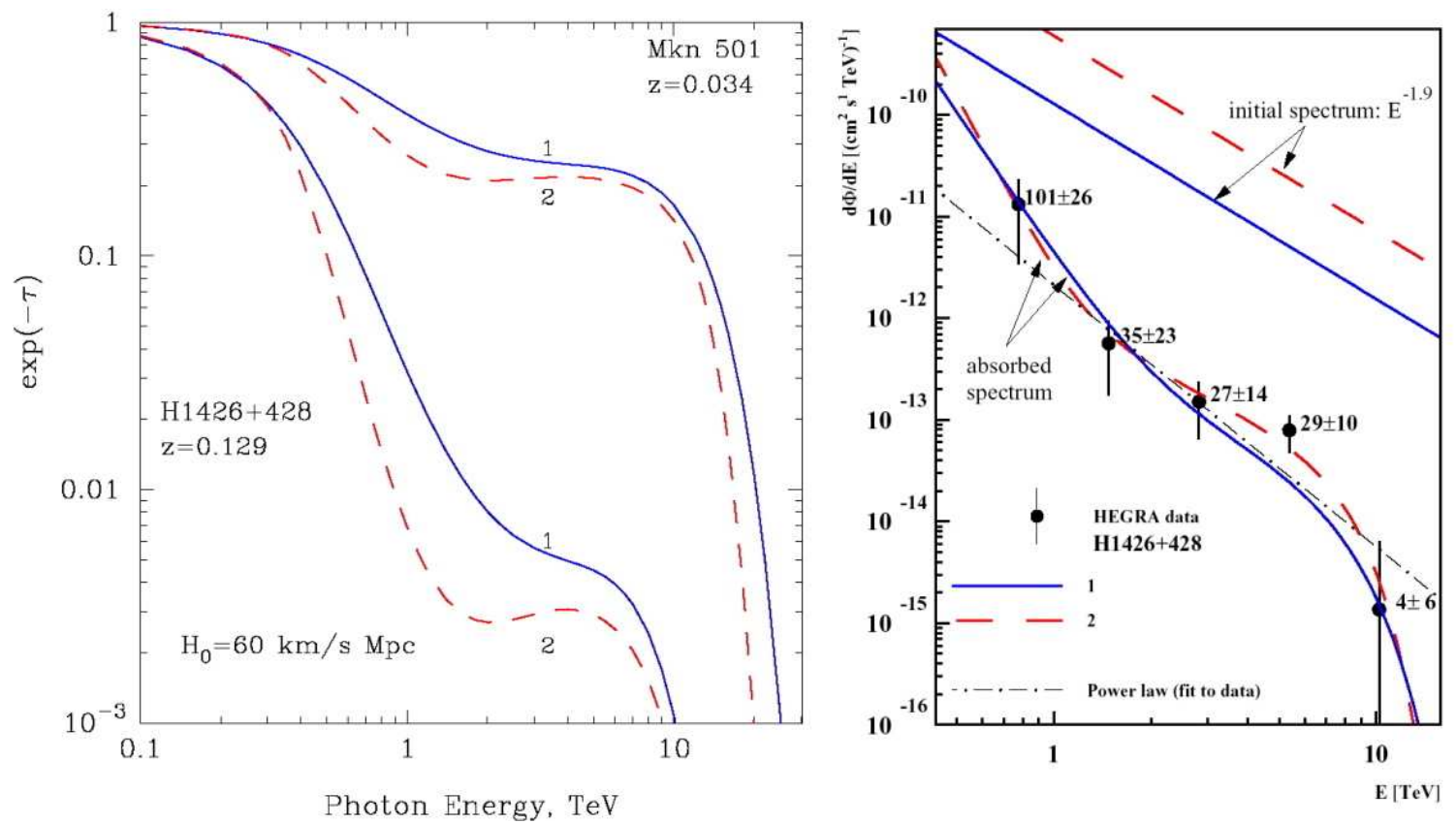

Figure 5: Left: The spectrum modification factors $\exp [-\tau(E)]$ calculated for the two infrared background models shown in figure 4 for Mkn 501 ( $z=0.034)$ and H 1426+428 ( $z=0.129)$. Right: differential energy spectrum of H 1426+428 as measured by [8] (dots); the numbers indicate the (background subtracted) event counts and their corresponding statistical $1 \sigma$-errors. A power law fit to the data is shown by the dasheddotted line. The solid and the dashed lines represent absorbed model spectra, obtained by an initial power law spectrum $\propto E^{-1.9}$ (normalized to the data) combined with different absorption models (see Fig. 4).

$\mathrm{TeV}$ blazars, according to which the synchrotron (X-ray) peak dominates over the inverse Compton $(\mathrm{TeV})$ peak (see e.g.[9]), unless we are in the presence of new effects that can modify the infrared absorption. One possibility is that quantum-gravity spacetime modification of Lorenz laws introduces an higher threshold condition. The primary challenge of quantum-gravity phenomenology is the one of establishing the properties of space-time at Planckian distance scales, since most theoretical arguments suggest that this is the characteristic scale of quantum space-time effects. However, there is also recent discussion of the possibility that quantum-spacetime effects might be stronger than usually expected, i.e. with a characteristic energy scale that is much smaller (perhaps in the $\mathrm{TeV}$ range) than the Planck energy. Examples of mechanisms leading to this possibility are found in string-theory models with large extra dimensions [10], in certain noncommutative-geometry models [11] and in other different frameworks [12]. Of course, the study of the phenomenology of these models is in the spirit of quantum-gravity phenomenology, but it has, in a sense, to be considered as a sideline development (and it is less challenging than the quantum-gravity-phenomenology efforts that pertain effects originating genuinely at the Planck scale).

This candidate quantum-gravity effect would be induced by a deformed dispersion relation for photons of the form [15]

$$
c^{2} \mathbf{p}^{2}=E^{2}\left[1+f\left(E / E_{\mathrm{QG}}\right)\right]
$$

where $E_{\mathrm{QG}}$ is an effective quantum-gravity energy scale and $f$ is a model-dependent function of the dimensionless ratio $E / E_{\mathrm{QG}}$. 


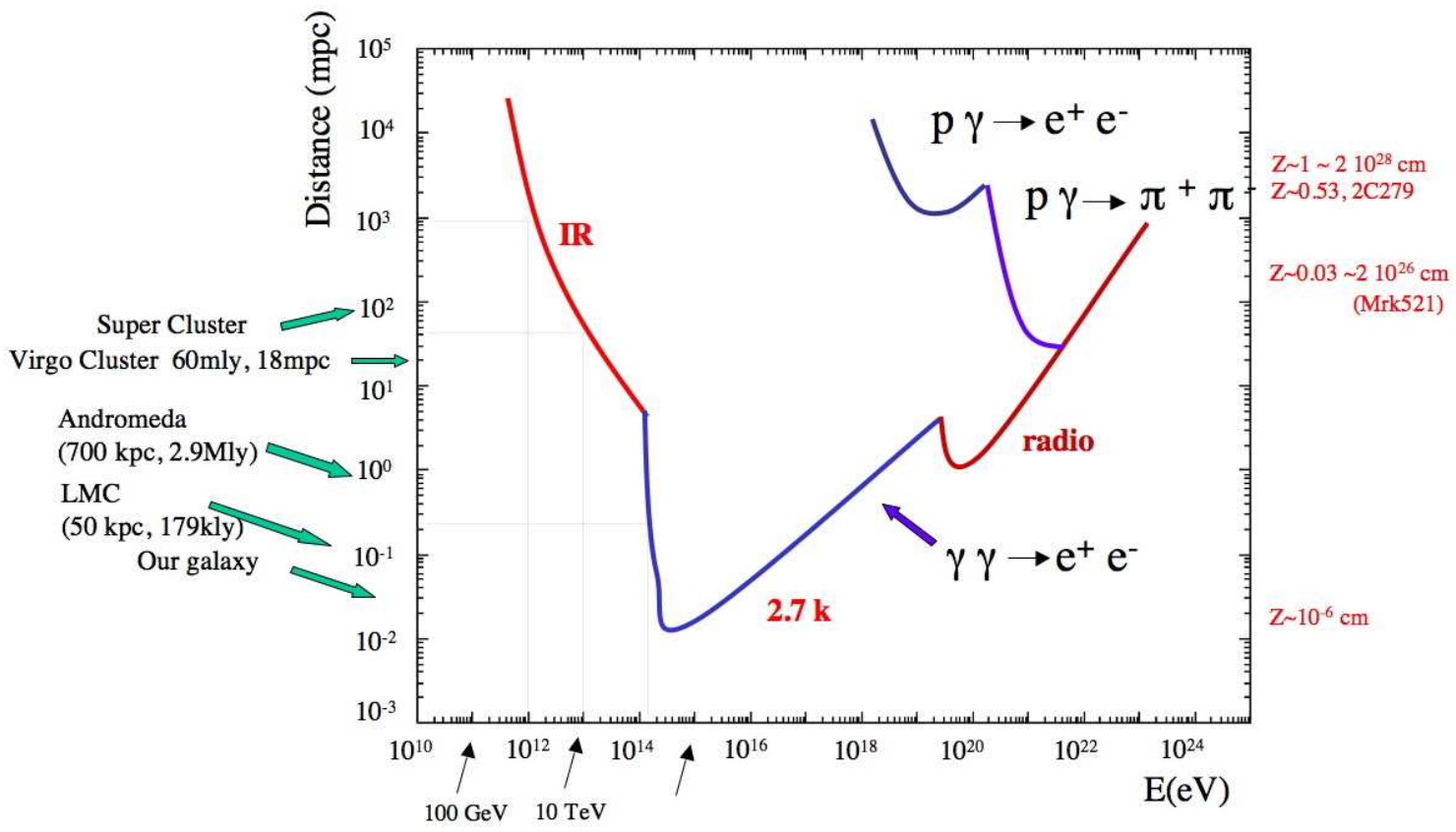

Figure 6: Transparency of the Universe due to the interaction with the infrared and microwave backgrounds: the mean free path for high energy -ray attenuation vs. energy.

The higher threshold condition introduced by quantum-gravity is [15]

$$
\varepsilon \geq \frac{m_{e}^{2}}{E}+\frac{E^{2}}{8 E_{Q G}}
$$

From the only hypothesis that we have seen some level of absorption in ref.[15] is derived a limit for $E_{Q G}$

$$
E_{Q G} \geq 2.6 \cdot 10^{14} \mathrm{TeV}
$$

Given that the observations still give us only a preliminary picture of absorption together and that there is a significant level of uncertainty in phenomenological models of $\mathrm{TeV}$ blazars and in phenomenological models of the density of the infrared diffuse extragalactic background, we are not allowed to convert these observations into tighter limits on departures from the classicalspacetime analysis However GLAST will dramatically extend the number of observed AGNs, as well as the energy range over which they can be observed. Indeed, GLAST might be called the "Hubble Telescope" of gamma-ray astronomy as it will be able to observe AGN sources to $\mathrm{z} \sim 4$ and beyond, if such objects actually existed at such early times in the universe. Extrapolation from EGRET AGN detections projects that about 5,000 AGN sources will be detected in a 2 year cumulative scanning mode observation by GLAST, as compared to the 85 that have been observed by EGRET in a similar time interval. This large number of AGN's covering a redshift range from $\mathrm{z}$ $\sim 0.03$ up to $\mathrm{z} \sim 4$ will allow to disentangle an intrinsic cutoff effect, (i.e., intrinsic to the source) from a cut-off derived from the interaction with the infrared background described earlier. Only by observing many examples of AGN, and over a wide range of redshifts, one can hope to untangle these two possible sources of cutoff. 


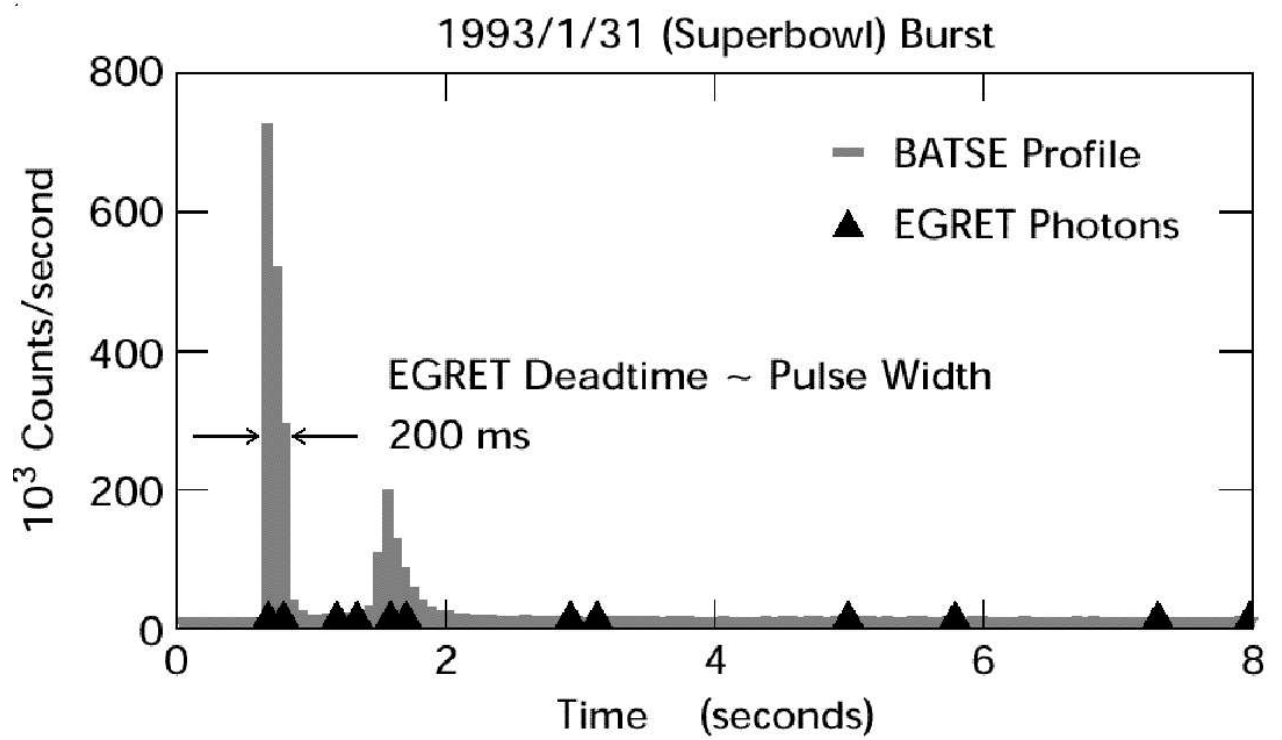

Figure 7: EGRET and BATSE light curves of the Superbowl burst, GRB930131. The burst consisted of an extremely intense spike, followed by low-level emission for several seconds. The true temporal development at energies $>100 \mathrm{MeV}$ is uncertain since EGRET dead time is comparable to GRB pulse widths.

\subsection{Gamma ray burst}

Gamma-ray bursts (GRBs) are intermittently the most intense and most distant known sources of high-energy gamma rays; at GeV energies, the brightest GRBs are 1000-10,000 times brighter than the brightest AGN. The unparalleled luminosities and cosmic distances of GRBs, combined with their extremely fast temporal variability, make GRBs an extremely powerful tool for probing fundamental physical processes and cosmic history. Figure 7 illustrates a very intense, short GRB. The true EGRET time profile is very uncertain because the $\sim$ two hundred milliseconds EGRET dead time per photon is comparable to GRB pulse widths; hence, many more photons may have been incident on EGRET during the extremely intense initial pulse. The GLAST dead time will be $\sim 10,000$ times smaller (see figure 2), thus allowing a precise measurement of the gamma-ray flux peak. This characteristic together with its larger field of view and larger effective area, should permit to detect virtually all GRBs in its field of view reaching "the edge" of the GRB distribution, as does BATSE. GLAST, with negligible self veto, will have good efficiency above $10 \mathrm{GeV}$ and it will be able to localize GRBs with sufficiently high accuracy to enable rapid searches at all longer wavelengths. About half of the 200 bursts per year detected by GLAST will be localized to better than 10 arc minute radius, an easily imaged field for large-aperture optical telescopes.

This will allow to study with an unprecedented precision the high energy time structure of transient events and to probe Quantum Gravity with the use of photons coming from an impulsive source looking for velocity dispersion laws, i.e. the velocity of light depends on the photon energy $E$ [16]. The basic idea is shown in figure 8 and it is a consequence of the same deformed dispersion relation of Eq. (3.2).

In quantum-gravity scenarios where the Hamiltonian equation of motion $\dot{x}_{i}=\partial H / \partial p_{i}$ is still valid (at least approximately) such a deformed dispersion relation would lead to energy-dependent 


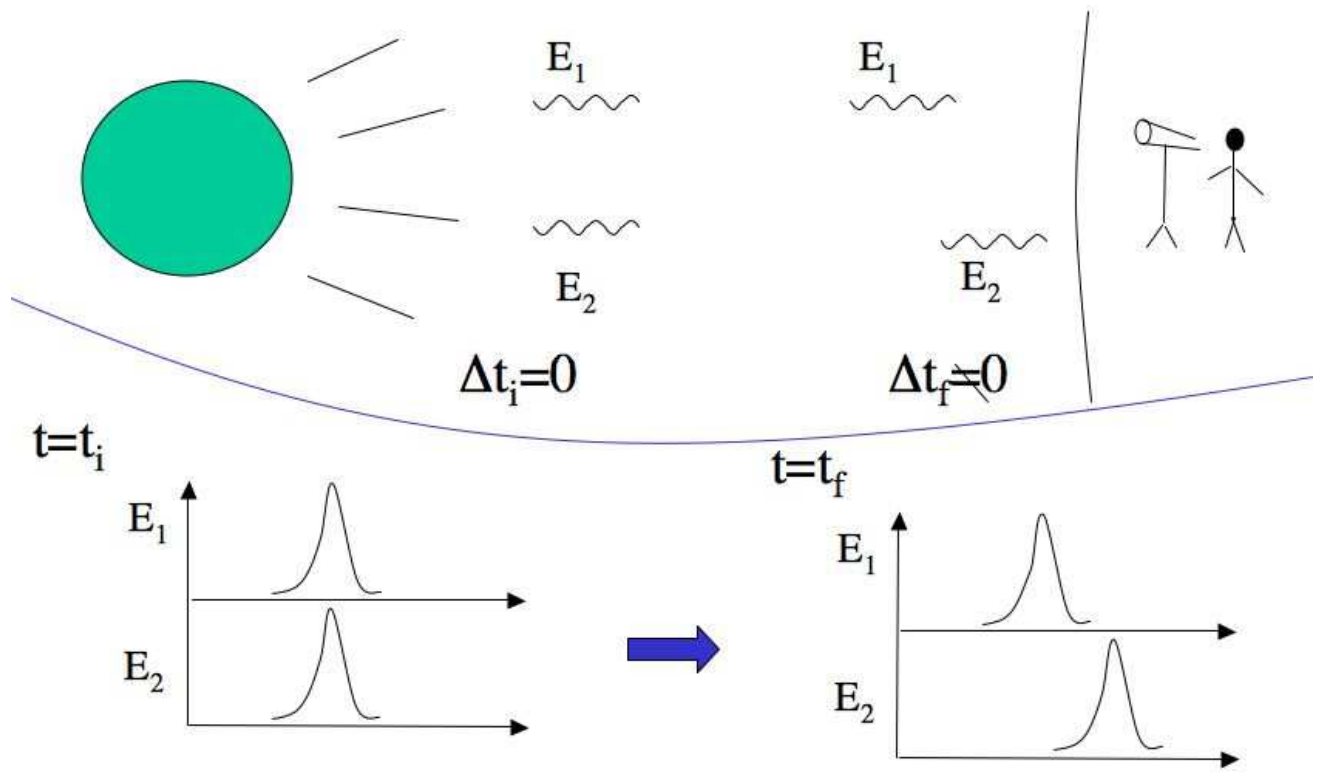

Figure 8: Simplified scheme for a Quantum Gravity Test with the use of photons coming from an impulsive source

velocities $c+\delta v$ for massless particles, with implications for all the electromagnetic signals that we receive from astrophysical objects at large distances. At small energies $E \ll E_{\mathrm{QG}}$, we expect that a series expansion of the dispersion relation should be applicable:

$$
c^{2} \mathbf{p}^{2}=E^{2}\left[1+\xi E / E_{\mathrm{QG}}+\mathscr{O}\left(E^{2} / E_{\mathrm{QG}}^{2}\right)\right]
$$

where $\xi= \pm 1$ is a sign ambiguity that depends on the dynamical framework and in certain models on the polarization state of the photon [13]. Such a series expansion would correspond to energydependent velocities

$$
v=\frac{\partial E}{\partial p} \sim c\left(1-\xi \frac{E}{E_{\mathrm{QG}}}\right)
$$

The idea is that the vacuum acts as quantum-gravitational medium which responds differently to the propagation of particle of different energies. (analogous to the propagation through an electromagnetic plasma).

We will not discuss the theoretical aspects of the problem, that can be found in [16] and references therein, but we will focus on the potentialities of AGILE and GLAST to probe this relation. Assuming that two photons, with different energies $E_{1}$ and $E_{2}$ are emitted at the same time, Eq.3.6 shows that they will arrive with a delay [14]

$$
\Delta t=\Delta E / E_{\mathrm{QG}} \cdot D / c
$$

For example for $z \sim 1 \sim D \sim 2 \cdot 10^{28} \mathrm{~cm}, E_{\mathrm{QG}} \sim 10^{19} \mathrm{GeV}$ we have $\Delta t(m s) \sim 60 \cdot \Delta E(\mathrm{GeV})$, that is a time interval in the domain of the future gamma ray experiments AGILE and GLAST. The 
MAGIC experiment has already set a limit $E_{\mathrm{QG}} \geq 0.2610^{18} \mathrm{GeV}$ with the observervation of a flare from Mkn501 [17]. With GLAST even at pulsars distance $D \sim 210^{28} \mathrm{~cm}$ the level of $E_{\mathrm{QG}}$ that can be tested is $E_{\mathrm{QG}} \sim 10^{14} \mathrm{GeV}$. Of course a strong assumption is required about the knowledge of the emission time of the photon from the source. However this knowledge can be bypassed if we will observe many source at different $z$ with GLAST.

It is important to stress that, in respect to the previous absorption case, by studying time-ofarrival/energy correlations in bursts of photons one is truly probing the kinematics of massless particles. This is a "clean probe" of Planck-scale kinematics because the absence of the particle mass constrains the roles that the Planck scale can play in kinematics.

Another possibility is to use the information provided by multiwave- length observations versus a full and self-consistent computation of the broad band spectrum of the Crab Nebula. A constraint of order $10^{-6}$ at $99 \% \mathrm{CL}$ on the on the lepton Lorentz Violation parameters can be achieved with GLAST [18].

We have discussed the capabilities of next generation gamma ray experiments to probe quantum gravity. In the following we will give a brief description of the experiments themselves.

\section{AGILE}

The techniques for the detection of gamma-rays in the pair production regime energy range are very different from the X-ray detection ones. Focusing is possible for X-rays detection and this permits large effective area, excellent energy resolution and very low background. For gammarays no focusing is possible and this means limited effective area, moderate energy resolution, high background but a wide field of view (see figure 10). The possibility to have a wide field of view is now enhanced, in respect to EGRET, with the use of silicon detectors. This new technique allows a further decrease of the ratio between height and width, essentially for two reasons: a) an increase of the position resolution that allows a decrease of the distance between the planes of the tracker without affecting the angular resolution, b) the possibility to use the silicon detectors themselves for the trigger of an event, with the elimination of the Time of Flight system, that requires some height. In figure 11 the basic principle and main elements of a pair conversion telescope is shown.

AGILE is a mission that will explore the gamma-ray Universe with a very innovative instrument, combining for the first time a gamma-ray imager (sensitive in the range $30 \mathrm{MeV}-50 \mathrm{GeV}$ ) and a hard X-ray imager (sensitive in the range 18-60 keV). An optimal angular resolution and very large field of view are obtained by the use of state-of-the-art silicon detectors integrated in a very compact instrument. AGILE was successfully launched on April 23, 2007 from the Indian base of Sriharikota (see figure 9) and was inserted in a low-particle background equatorial orbit.

The AGILE design was derived from a study of the GILDA project [19],[20] that was based on the techniques developed for the WiZard silicon calorimeter [21], successfully flown in balloon experiments.

A comparison between AGILE and the most famous previous gamma-ray satellites is shown in fig.12. The dimensions of AGILE are comparable to those of SAS-2 and Cos-B, but thanks to the advance of the techniques the performances of AGILE are better than that of EGRET.

AGILE is supported by the Italian Space Agency (ASI) with scientific and programmatic participation by INFN, INAF and several Italian universities. 


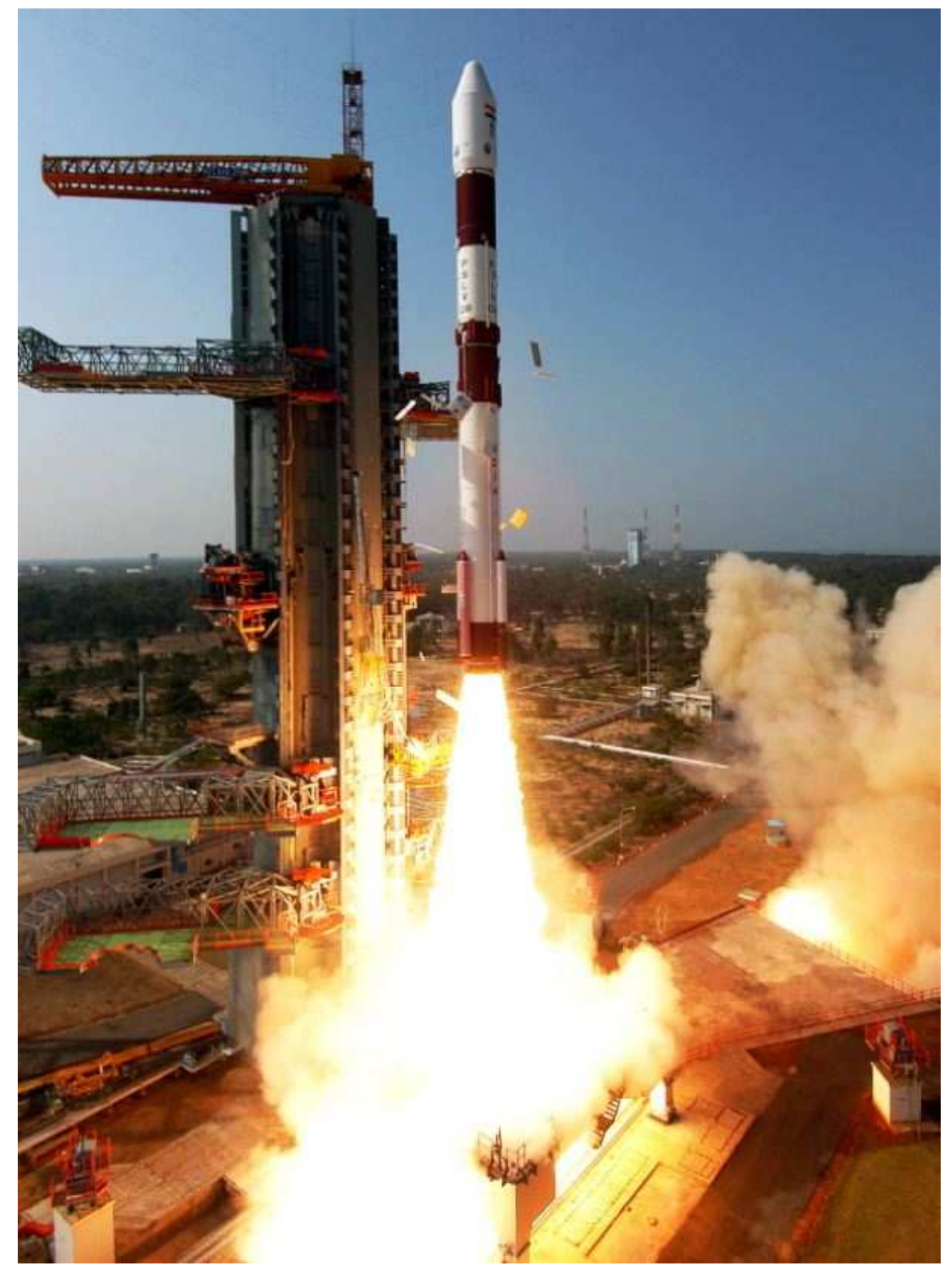

Figure 9: The launch of the AGILE satellite by the Indian PSLV-C8 rocket from the Sriharikota base on April 23, 2007.

The names of the people involved in the collaboration together with the on-line status of the project are available at http://people.roma2.infn.it/ agile/.

AGILE instrument total weight is $\sim 80 \mathrm{~kg}$ including the Si-Tracker, Super-AGILE, MiniCalorimeter, the Anticoincidence system and electronics. A description of the capabilities of AGILE can be found in [22].

\section{GLAST}

The Gamma-ray Large Area Space Telescope (GLAST) [23] has been selected by NASA as a mission involving an international collaboration of particle physics and astrophysics communities from the United States, Italy, Japan, France and Germany for a launch in the first half of 2008.

The main scientific objects are the study of all gamma ray sources such as blazars, gamma-ray bursts, supernova remnants, pulsars, diffuse radiation, and unidentified high-energy sources. Many 


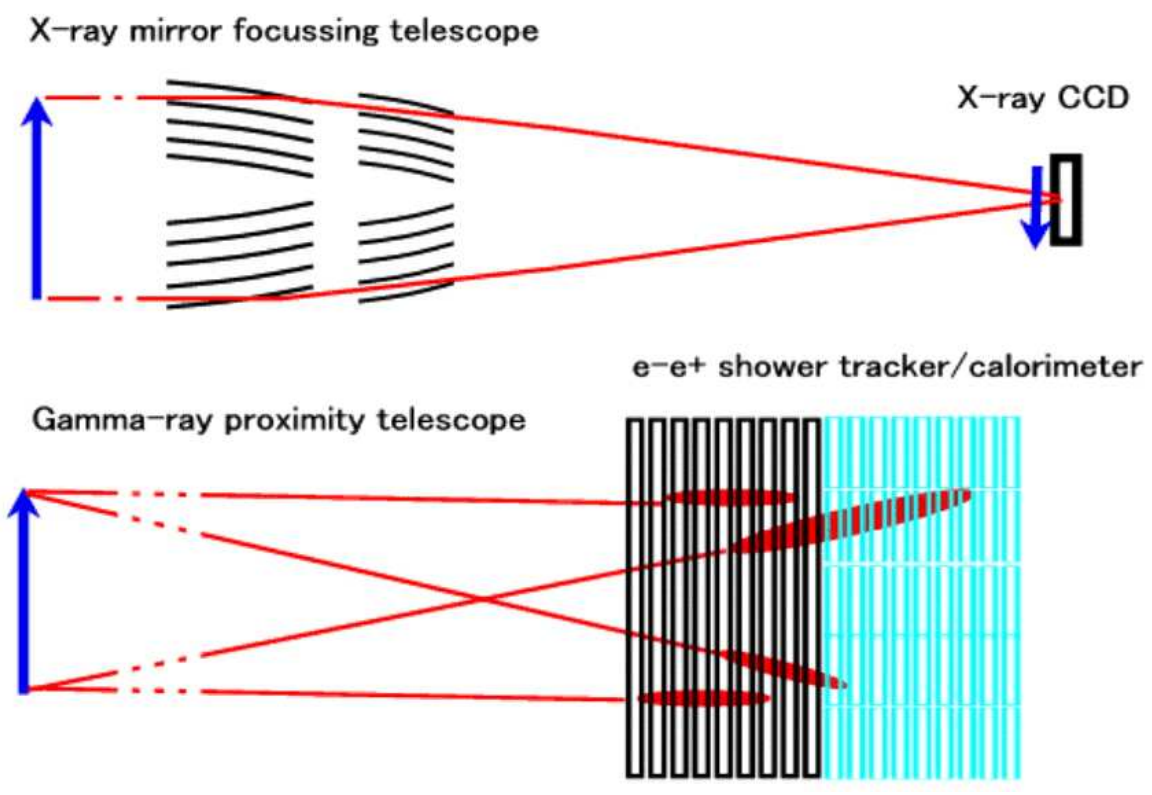

Figure 10: Detector Technology: X-ray versus Gamma-ray

years of refinement has led to the configuration of the instrument shown in figure 13, where one can see the $4 \mathrm{x} 4$ array of identical towers each formed by: • Si-strip Tracker Detectors and converters arranged in $18 \mathrm{XY}$ tracking planes for the measurement of the photon direction. • Segmented array of $\mathrm{CsI}(\mathrm{Tl})$ crystals for the photon energy measurement. - Segmented Anticoincidence Detector (ACD). The main characteristics, shown in figure 14, are an energy range between $20 \mathrm{MeV}$ and $300 \mathrm{GeV}$, a field of view of $\sim 3 \mathrm{sr}$, an energy resolution of $\sim 5 \%$ at $1 \mathrm{GeV}$, a point source sensitivity of $2 \times 10^{-9}\left(\mathrm{ph} \mathrm{cm}^{-2} \mathrm{~s}^{-1}\right)$ at $0.1 \mathrm{GeV}$, an event deadtime of $20 \mu \mathrm{s}$ and a peak effective area of 10000 $\mathrm{cm}^{2}$, for a required power of $600 \mathrm{~W}$ and a payload weight of $3000 \mathrm{Kg}$.

The list of the people and the Institution involved in the collaboration together with the on-line status of the project is available at http://www-glast.stanford.edu.

GLAST could also be of particular interest for the search of dark matter candidates. If dark matter is made by the lightest supersymmetric particles (neutralinos), they would have nonrelativistic velocities; hence the neutralino annihilation into two $\gamma^{\prime}$ 's and a $\gamma$ and a $\mathrm{Z}$ as final states can give rise to $\gamma$-rays with unique energies $E_{\gamma}=M_{\chi}$ and $E_{\gamma}^{\prime}=M_{\chi}\left(1-m_{z}^{2} / 4 M_{\chi}^{2}\right)$. All the others annihilation process will give also $\pi^{0}$ that will decay in a continuum gamma-ray flux. Studies has been carried out [24, 25] and are under way [27] to show that in this way a large number of supersymmetric models can be tested.

This effort will be complementary to a similar search for neutralinos looking with cosmicray experiments like the next space experiments PAMELA[28] and AMS[29] at the distortion of the secondary positron fraction and secondary antiproton flux induced by a signal from a heavy neutralino.

A number of space experiments are under construction. For X-ray and gamma-ray experiments the time of operation versus energy range is shown in figure 15. Note that AGILE and GLAST cover an interval not covered by any other experiments. Note also the number of other experiments 


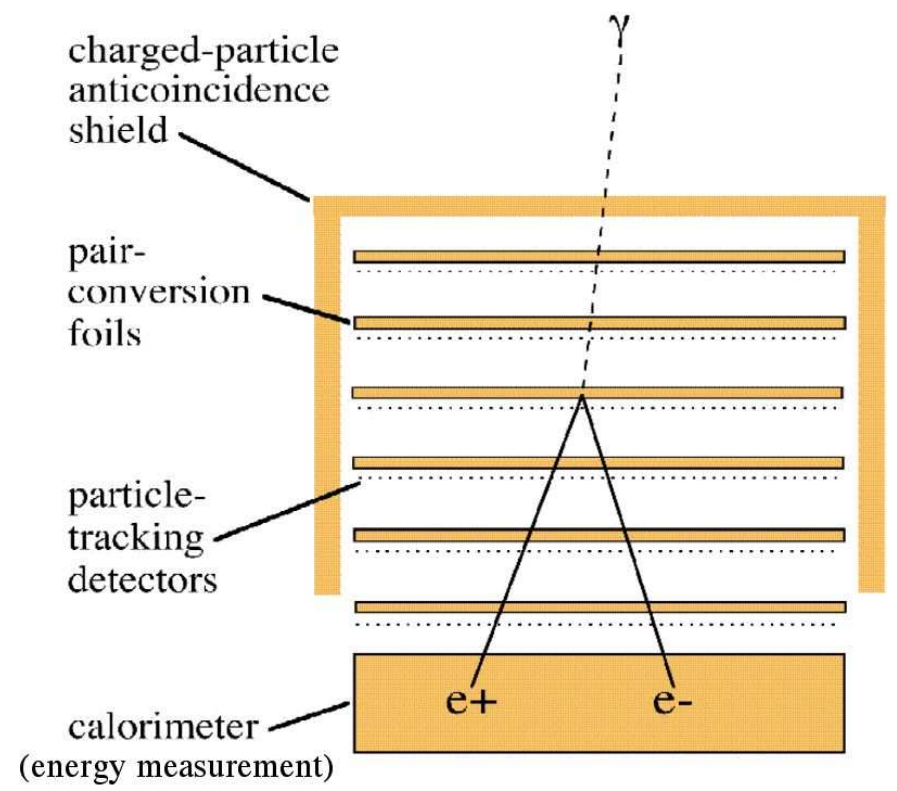

Figure 11: Main elements of a pair conversion telescope and principle of detection

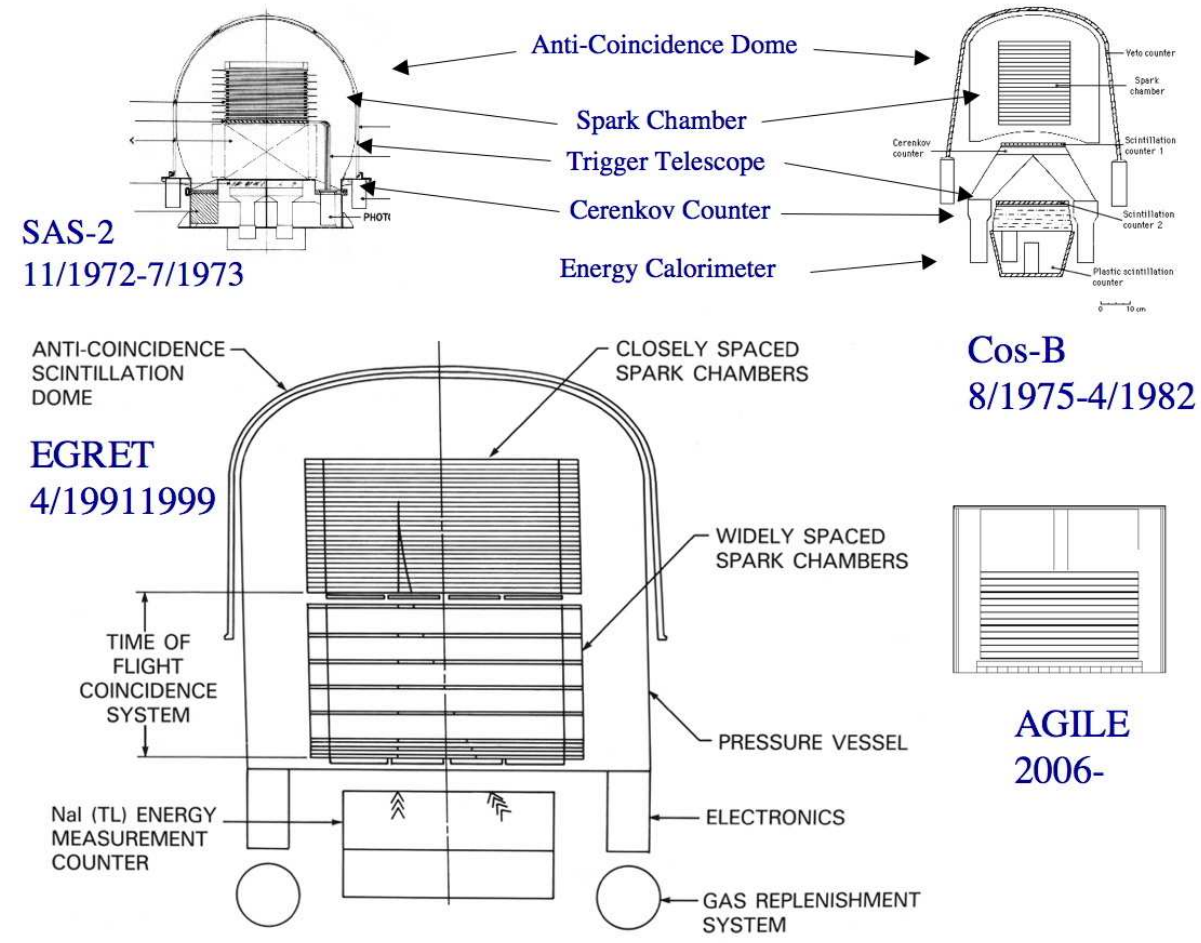

Figure 12: Comparison between the paste gamma ray experiments (SAS-2, Cos-B, EGRET) and AGILE. 


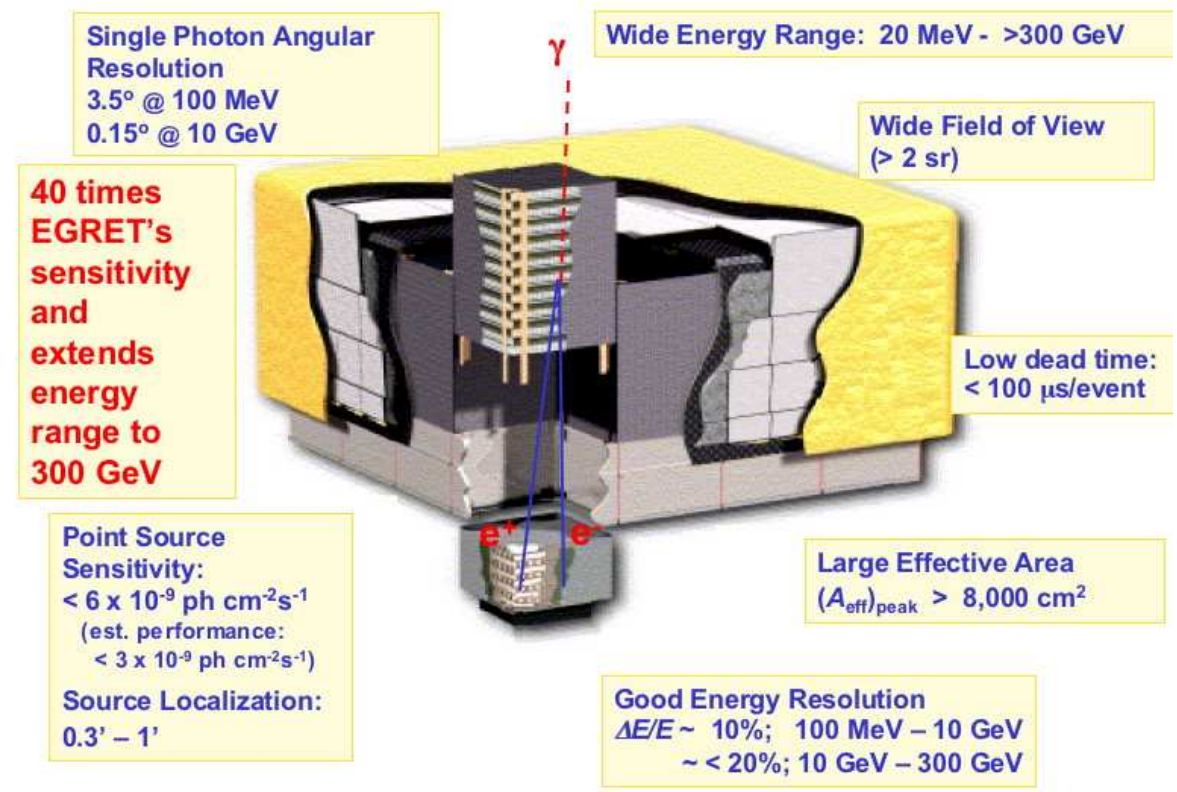

Figure 13: The GLAST instrument, exploded to show the detector layers in a tower, the stacking of the CsI logs in the calorimeter, and the integration of the subsystems.
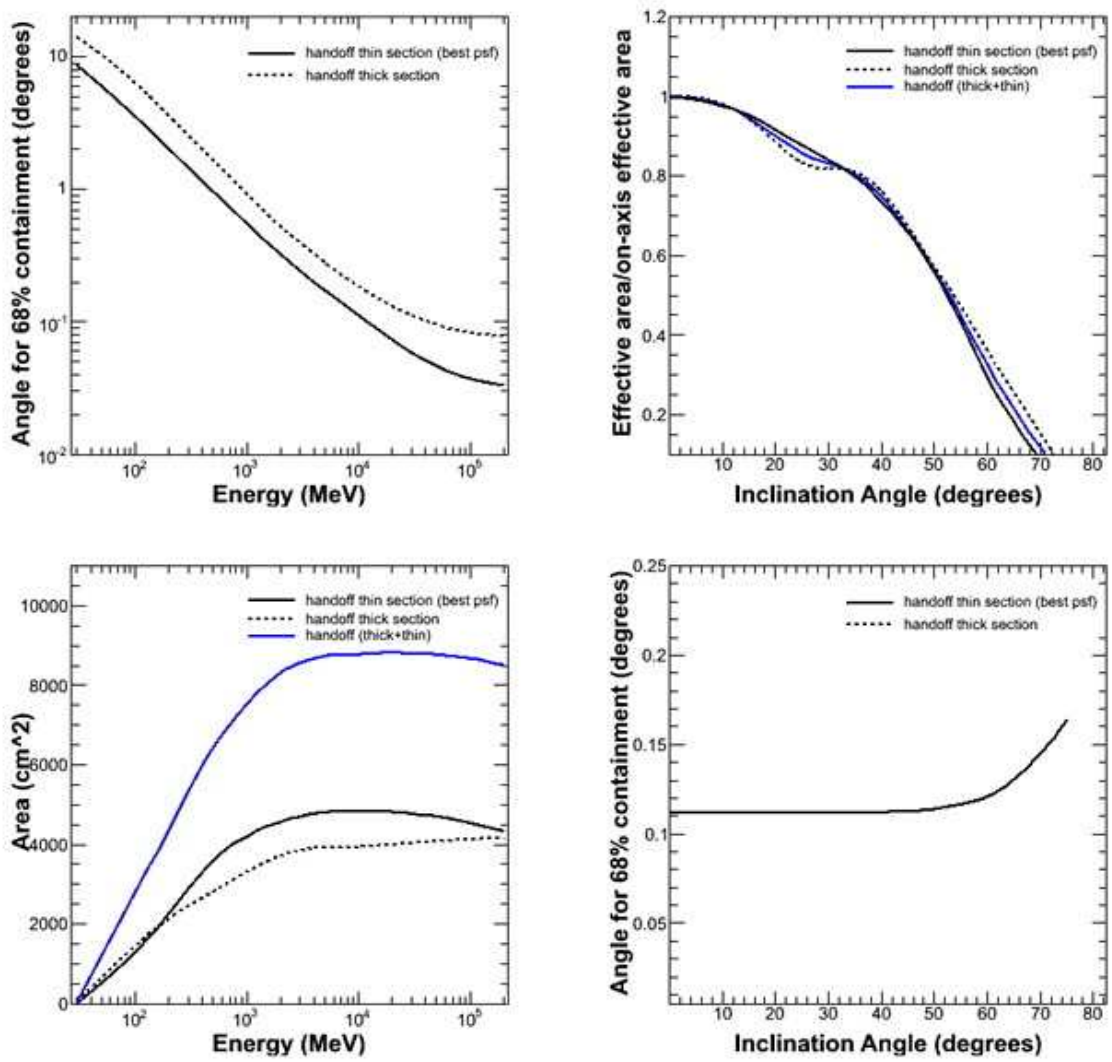

Figure 14: GLAST instrument performance, including all background and track quality cuts [26] 


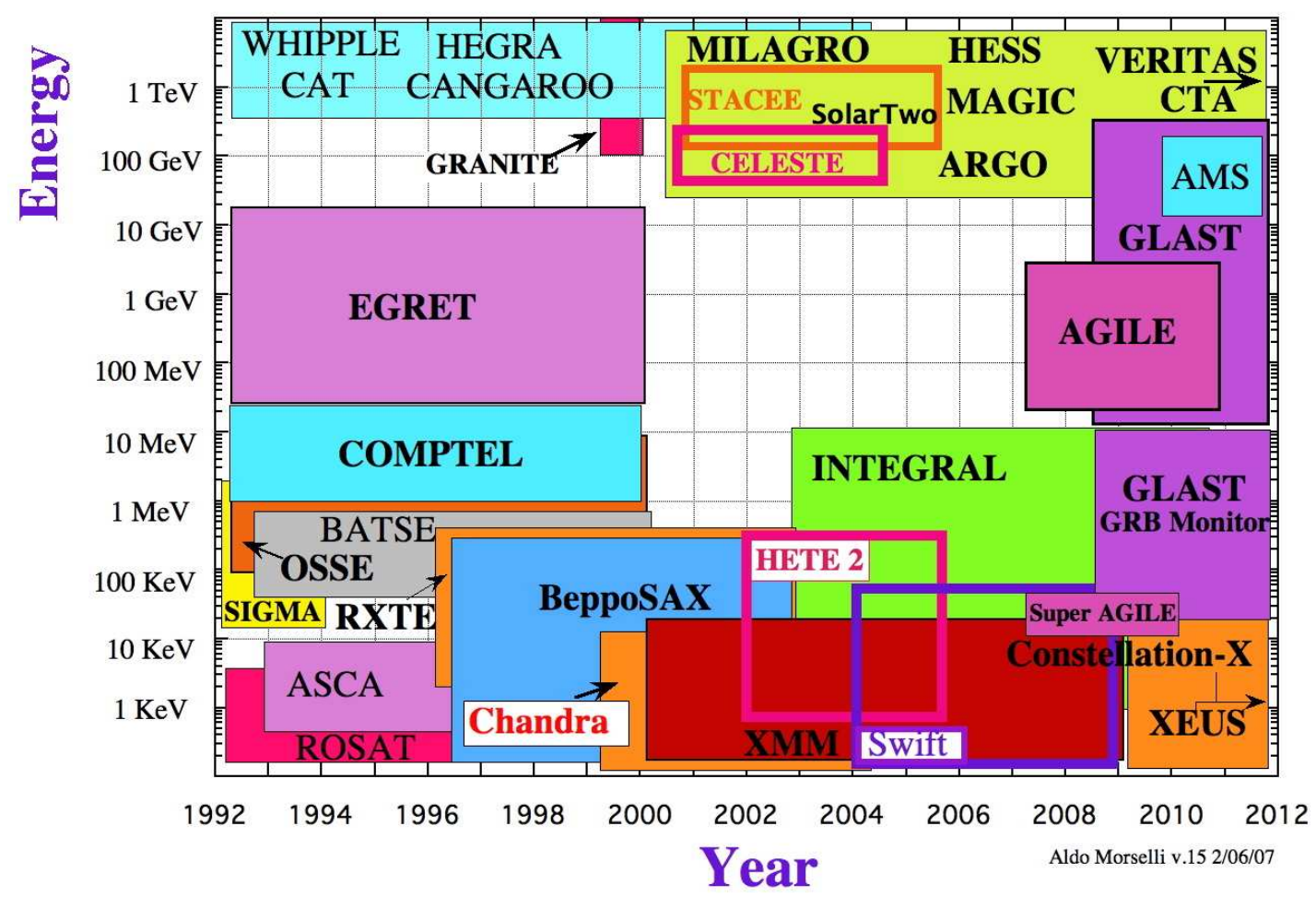

Figure 15: Energy versus time of gamma-ray experiments

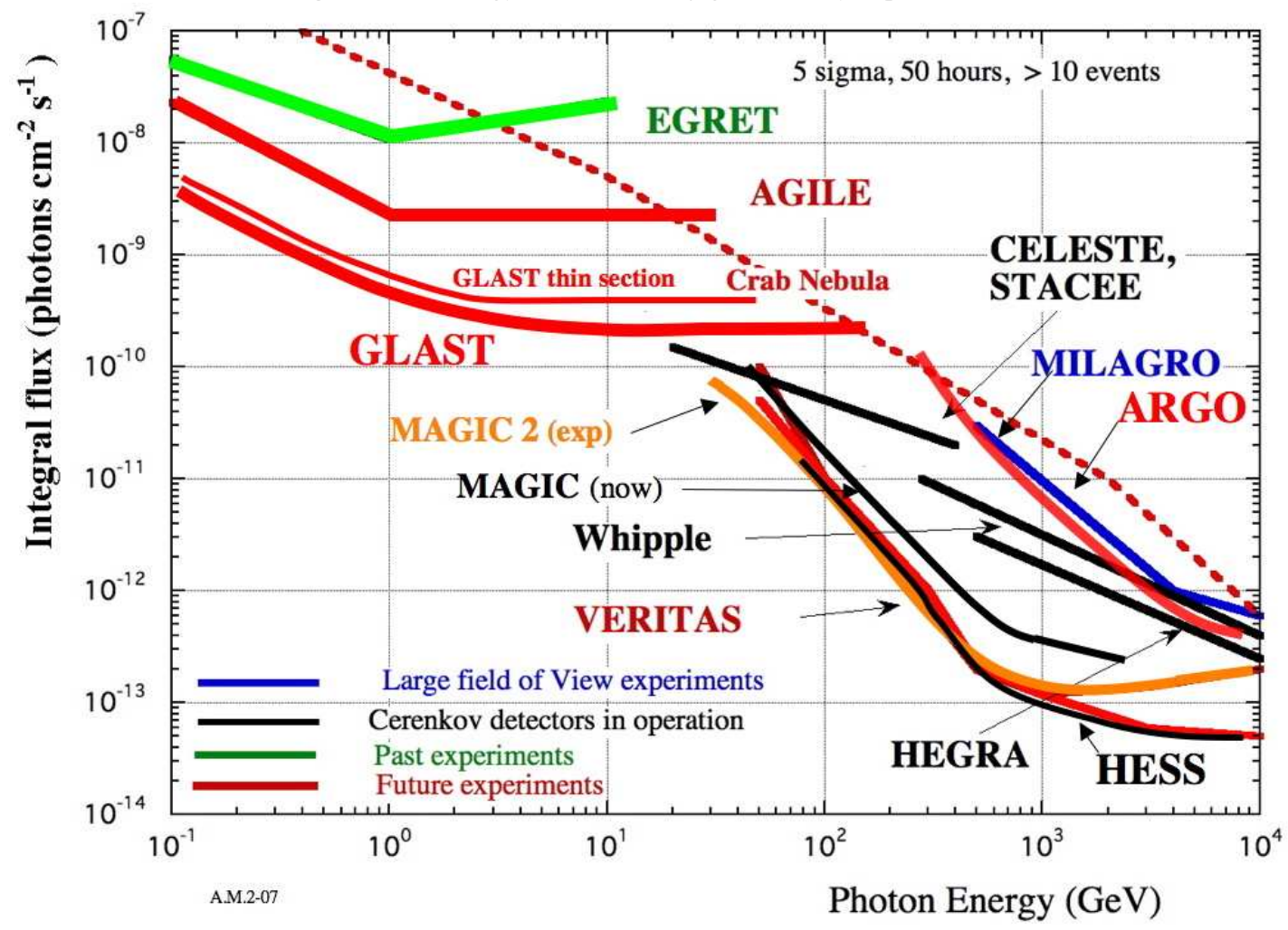

Figure 16: Sensitivity of $\gamma$-ray detectors 
at other frequencies that will allow extensive multifrequency studies.

In the last decade, ground-based instruments have made great progress, both in technical and scientific terms. High-energy gamma rays can be observed from the ground by experiments that detect the air showers produced in the upper atmosphere. Air shower arrays directly detect the particles (electrons, muons, and photons) in air showers, and atmospheric Cerenkov telescopes detect the Cherenkov radiation created in the atmosphere and beamed to the ground. Detectors based on the atmospheric Cerenkov technique consist of one or more mirrors that concentrate the Cerenkov photons onto fast optical detectors. Photomultiplier tubes (PMTs) placed in the focal plane are generally used to detect the Cherenkov photons. Two problems in using atmospheric Cherenkov telescopes (ACT) are the night-sky background and the large isotropic background from cosmic-ray showers.

\section{Ulimate Objective: To image the particle accelerator near the event}

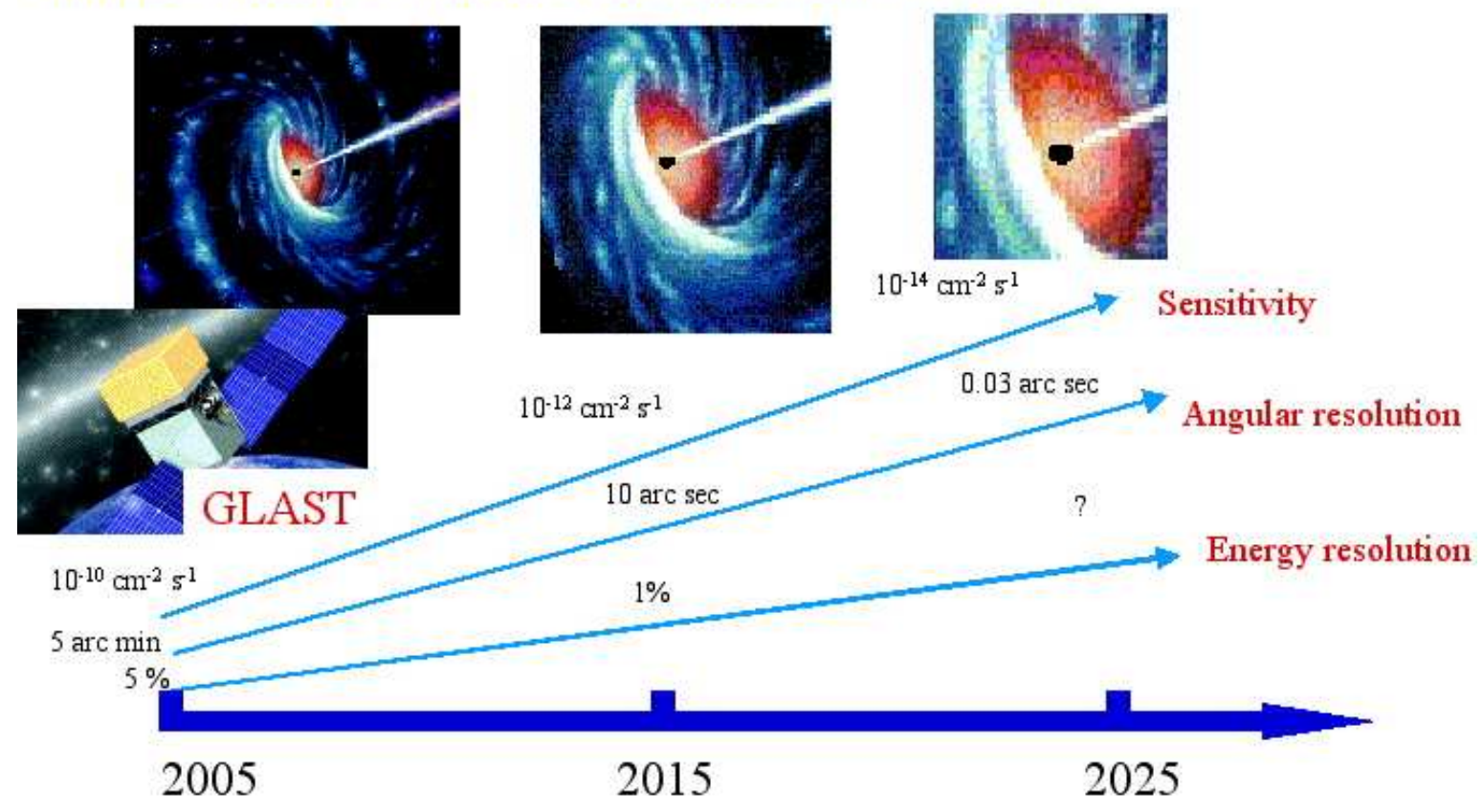

Figure 17: Gamma-Ray Astronomy Long Term Plan

The energy threshold of an atmospheric Cherenkov telescope is determined by the number of Cherenkov photons needed to observe a signal above the level of the night-sky background. For individual point sources, ground-based instruments have unparalleled sensitivity at very high energies (above 50-250 GeV). For many objects, full multi-wave-length coverage over as wide an energy range as possible will be needed to understand the acceleration and gamma-ray production mechanisms. On the technical side, atmospheric Cherenkov telescopes have demonstrated that a high degree of gamma/hadron discrimination and a source pointing accuracy of 10-30 arc minutes (depending on the source strength) can be achieved based on the detected Cherenkov image. Also the energy threshold is lowering remarkably. GLAST sensitivity compared with other present and future detectors in the gamma-ray astrophysics range is shown in figure 16. The predicted sensitivity of a number of operational and proposed Ground based Cherenkov telescopes, 


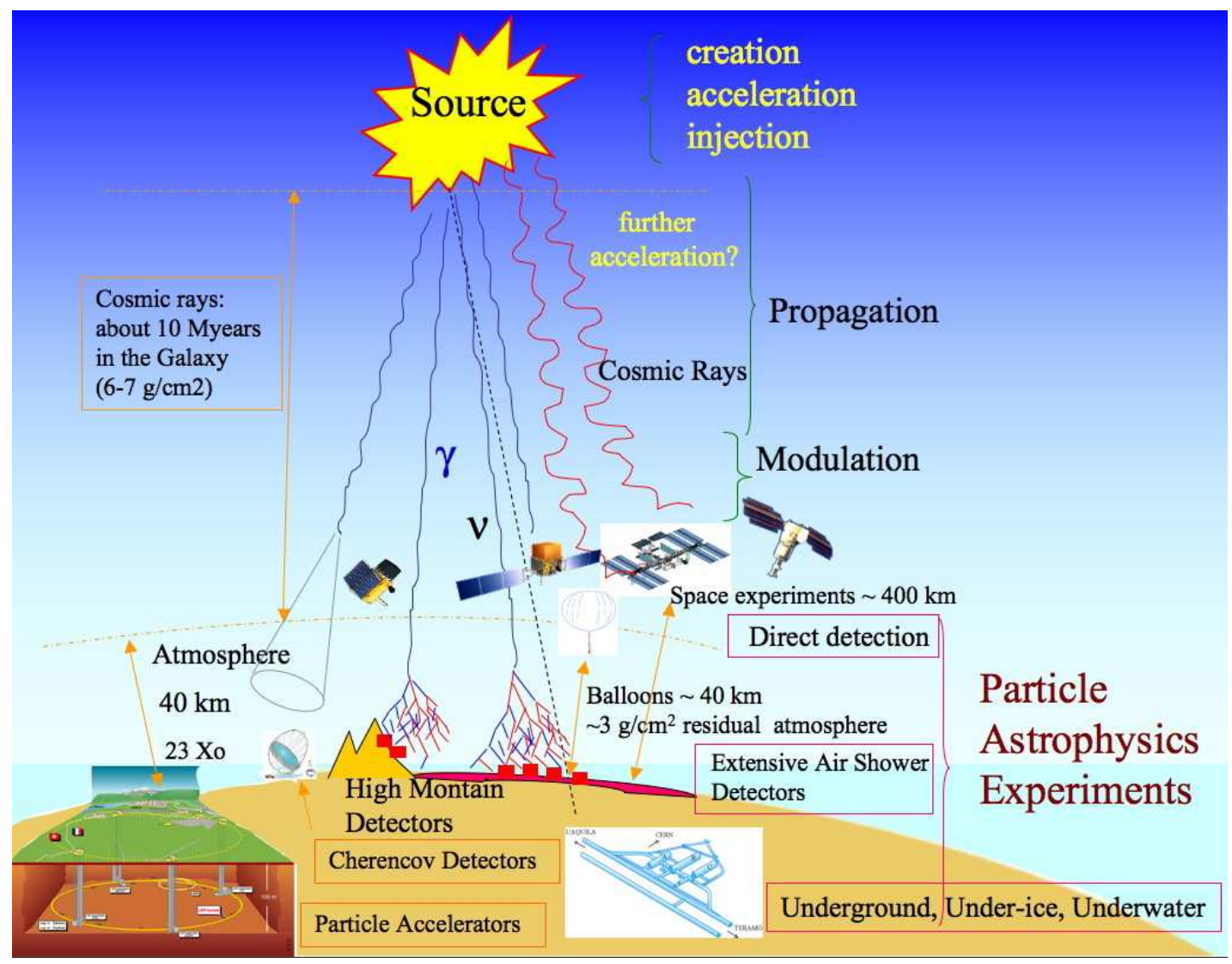

Figure 18: Particle Astrophysics

CELESTE, STACEE, VERITAS, Whipple, HESS, MAGIC, is for a 50 hour exposure on a single source. EGRET, GLAST, MILAGRO, ARGO and AGILE sensitivity is shown for one year of all sky survey. The diffuse background assumed is $2 \cdot 10^{-5}$ photons $\mathrm{cm}^{-2} \mathrm{~s}^{-1} \mathrm{sr}^{-1}(100 \mathrm{MeV} / E)^{1.1}$, typical of the background seen by EGRET at high galactic latitudes. The source differential photon number spectrum is assumed to have a power law index of -2, typical of many of the sources observed by EGRET and the sensitivity is based on the requirement that the number of detected source photons is at least 5 sigma above the background. Note that on ground only MILAGRO and ARGO will observe more than one source simultaneously.

It is worth to note that the angular resolution and energy resolution achievable in gamma ray astrophysics is still lower to what is desirable and achievable in other band; so a long term plan like the one sketched in figure 17 is needed and can bring spectacular results.

We have tried to describe the activities in space experiments and their correlation with quantum gravity test and dark matter search. Space particle astrophysics is becoming a reality and together with the development of the extensive air shower detectors and the underground detectors will give a big improvement in our understanding of the laws of the Universe (see figure 18)

\section{References}

[1] A.Harding, Proc. XXXVIIth Rencontres de Moriond "The Gamma-Ray Universe", astro-ph/0208421 
[2] J.Daugherty and A.Harding, 1996, Astophys. Journal 458278.

[3] R.Romani, 1996, Astophys. Journal 470469.

[4] S. Johnston et al., High time resolution observations of the Vela pulsar, Astrophys J , 549, L101, 2001, M. Kramer et al., astro-ph/0203126

[5] L.D.Landau and E.M.Lifshist, Relativistic Quantum Theory, Mir, Moscow, 1965

[6] F.Aharonian et al. (HESS Collaboration) Nature, Vol.440, pp.1018, 2006

[7] Hauser, M. \& Dwek, E. 2001, Annual Reviews of Astronomy and Astrophysics, 39, 249, [astro-ph/0105539]

[8] F. Aharonian et.al, astro/ph 0202072

[9] Fossati, G., Maraschi, L., Celotti, A., et al. 1998, MNRAS, 299, 433.

[10] N. Arkani-Hamed, S. Dimopoulos and G. Dvali, Phys. Lett. B 429, 263 (1998).

[11] M. R. Douglas and N. A. Nekrasov, hep-th/0106048.

[12] V. A. Kostelecky and S. Samuel, Phys. Rev. D 39, 683 (1989)

R. Gambini and J. Pullin, Phys. Rev. D 59, 124021 (1999) [arXiv:gr-qc/9809038]

R.C. Myers and M. Pospelov, Phys. Rev. Lett. 90, 211601 (2003) [arXiv:hep-ph/0301124]

[13] T. Jacobson, S. Liberati, D. Mattingly, astro-ph/0505267

[14] J. Ellis et al, astro-ph/0510172, erratum arXiv:0712.2781

[15] G. Amelino-Camelia, gr-qc/0212002

[16] G. Amelino-Camelia, et al. Nature 393, 763 (1998) [astro-ph/9712103]

[17] J. Albert et al., arXiv:0708.2889

[18] L. Maccione, S. Liberati, A. Celotti, J. Kirk, , Science with the new generation high energy gammaray experiments, Frascati Physics Series Vol. 45 (2007) pp.243, edited by A.Lionetto and A. Morselli http://www.roma2.infn.it/SciNeGHE07/scineghe07_proc.pdf

[19] G. Barbiellini et al., Nucl. Inst. and Met. A354 (1995) 547

[20] A. Morselli et al., XXIV Int. Cosmic Ray Conf., OG 10.3.26, 3 (1995) 669, Roma

[21] P. Spillantini et al., Il Nuovo Cimento 103B (1989) 625

[22] A. Morselli et al., XXVI ICRC, OG 4.2.6, (1999) Salt Lake City

A. Morselli et al., Nuclear Physics B 85, (2000) 22

M. Tavani et al. (AGILE Collaboration), Science with the new generation high energy gamma- ray experiments, Frascati Physics Series Vol. 45 (2007) pp.1, edited by A.Lionetto and A. Morselli http://www.roma2.infn.it/SciNeGHE07/scineghe07_proc.pdf

[23] A. Morselli 1997, XXXIInd Rencontres de Moriond, Very High Energy Phenomena in the Universe, Les Arc, France, January 18-25, 1997, Editions Frontiers, p.123

W.B. Atwood et al, NIM A 583 (2007) 9

P.Michelson et al, First GLAST Symposium, Stanford, 2007, AIP CP921, 2007

[24] A.Morselli et al., Nuclear Physics B 113B (2002) 213-220

[25] A.Cesarini, F.Fucito, A.Lionetto, A.Morselli and P.Ullio, Astropart. Phys. 21 (2004) 267

[astro-ph/0305075] 
[26] http://www-glast.slac.stanford.edu/software/IS/glast_lat_performance.htm

[27] A. Morselli, G. Bertone, G. Busetto, R. Rando, T. Bringmann, on the behalf of the GLAST Collaboration, First GLAST Symposium, Stanford, 2007, AIP CP921, 2007, pg.506-507

[28] P. Picozza et al., (PAMELA Collaboration) Astroparticle Physics 27 (2007) 296

A.Lionetto, A.Morselli, V.Zdravkovic (2005), J. Cosmol. Astropart. Phys. JCAP09, 010 [astro-ph/0502406]

[29] S. Di Falco et al. (AMS Collaboration), Science with the new generation high energy gamma- ray experiments, Frascati Physics Series Vol. 45 (2007) pp.193, edited by A.Lionetto and A. Morselli http://www.roma2.infn.it/SciNeGHE07/scineghe07_proc.pdf 